\title{
Ultrasound simulation of peripheral nerves: development of a novel technology for training in regional anaesthesia
}

\author{
Raoul Breitkreutz $\cdot$ Sebastian Schellhaas \\ Thomas Schmitz-Rixen · Paul Kessler · \\ Felix Walcher
}

Published online: 26 September 2009

(C) Springer-Verlag 2009

\begin{abstract}
Objective Regarding education in ultrasound-guided regional anaesthesia, we aimed to develop three-dimensional ultrasound volumes (3D-UV) of peripheral nerves for ultrasound simulation. This study mainly addressed to estimate diagnostic feasibility as well as comparison of original ultrasound images with 3D-UV.

Methods A free-hand recording system as part of an ultrasound simulation system (Ultrasound simulator Reslice, Schallware GmbH, Berlin, Germany) was coupled to a high-end ultrasound device (Vivid-i, GE healthcare Ultrasound). 3D-UV were recorded as transverse parallel planes from four healthy models and positioned with an electromagnetic tracking system into a mannequin on identical anatomical sites. Using developer software, single B-Mode 3D-UV were combined into 3D-multivolumes (MV). Original short and long axis B-Mode planes were compared with reconstructed planes (reslices).
\end{abstract}

R. Breitkreutz $(\bowtie) \cdot S$. Schellhaas

Clinics of Anaesthesiology, Intensive Care Medicine and Pain

Therapy, Hospital of the Johann Wolfgang Goethe-University,

Frankfurt am Main, Germany

e-mail: raoul.breitkreutz@gmail.com

T. Schmitz-Rixen

Clinics of Vascular and Endovascular Surgery,

Hospital of the Johann Wolfgang Goethe-University,

Frankfurt am Main, Germany

P. Kessler

Department of Anaesthesiology, Intensive Care and Pain

Medicine, Orthopedic University Hospital Friedrichsheim

Foundation, Frankfurt am Main, Germany

F. Walcher

Department of Trauma Surgery, Hospital of the Johann

Wolfgang Goethe-University, Frankfurt am Main, Germany
Results A total of 108 3D-UV including vessels of carotid region $(n=22)$, and peripheral nerves of interscalene (31), axillary (28), femoral groin (8) and distal sciatic (19) were recorded. Visual presentation of reconstructed volumes of vessels and peripheral nerves revealed almost comparable quality to the original B-Mode scans of the model examination. Ultrasonographic nerve structure appearance derived from high-resolution ultrasound recordings could be preserved in reslices of 3D-UV and also in part in the long axis.

Conclusions Our data provide a new proof of concept for ultrasound simulation of peripheral nerves in 3D-ultrasound multivolumes based on high-resolution ultrasound technology. It has the ability to provide pathologic findings and may serve as a powerful method for training in regional anaesthesia and critical care ultrasound.

Keywords Ultrasound - Regional anaesthesia . Peripheral nerves · Education · Training · Critical care · Diagnostic $\cdot$ Feasibility

\section{Introduction}

Ultrasound-guided regional anaesthesia (UGRA) of peripheral nerves is an emerging method in anaesthesia and critical care environment. The basis of understanding this technique has to be firm knowledge of anatomy, topology and moreover of sonoanatomy. Identifying key landmark structures including blood vessels, muscle, fascia and bone, nerve and plexus on short axis (SAX) imaging, anatomy and anatomic variations were defined as the first three important tasks out of ten by current recommendations for education and training in UGRA only recently [1]. 
Unfortunately, there are only few methods of training to obtain skills for this technique. Normally it requires multiple observations and patient examinations in the operation room to understand sonoanatomy and procedural aspects and "learning by doing". Moreover, to get experience in anatomical variations or pathologic findings, a large series of patients have to be passed. Lectures and video clips can partially support this gap as they present only two-dimensional aspects of typical planes' sequence of events and do not allow a real time examination. Only few methods are available to train eye-hand coordination, e.g. by self-made organic physical trainings models [2]. However, to the best of our knowledge until now, a method for 3D ultrasound teaching in UGRA was unknown.

Ultrasound simulation was developed as a new tool for the focused abdominal sonography in trauma (FAST) to train the combination of handling an ultrasound probe and performing a real time examination with direct interpretation of ultrasound images. Thereby training was more independent of models and patients and also could address real-life scenarios within training courses. Furthermore, ultrasound simulation is of strong interest, because it can provide a large case numbers and thus provide teaching on anatomical variations and pathology [3-5].

Therefore, we hypothesized, whether this technology can also be exploited for high-resolution ultrasonography of peripheral nerves and aimed to develop 3D ultrasound volumes (3D-UV) of peripheral nerves for ultrasound simulation. This study therefore mainly addressed to estimate feasibility as well as comparison of original ultrasound images with 3D-UV.

\section{Methods}

Ultrasound simulator and recording system

A free-hand recording system as part of an ultrasound simulation system (Ultrasound simulator Reslice, registered trademark by Schallware GmbH, Berlin, Germany, http://www.schallware.de) [5] was coupled to a high-end ultrasound device (Vivid-i, registered trademark by GE Healthcare Ultrasound, Solingen, Germany) with a $12 \mathrm{MHz}$ linear probe (12L). The free-hand recording system consists of a PC with Ubuntu software platform (Linux-based public license, non-commercial software, version 7.10, http://www.ubuntu.com), an electromagnetic tracking system, a mannequin and a probe dummy with localization transmitter, and also consists of a PC with recording software (Schallware Acquisition 2007, Fa. Schallware, Berlin, Germany), and an additional electromagnetic tracking system. The ultrasound device was also coupled with a VGA2USB-LR converter (Epiphan Company, Springfield, NJ, USA) to get a lossless video-real time compression with high frame rates from up to 80 frames per second (fps). To save position of the probe of the ultrasound device once, the probe had to be connected and calibrated with a localization transmitter. Calibration of the probe had only to be done once before the beginning of the trial by the manufacturer of the ultrasound simulator. Frame rates while recording $3 \mathrm{D}-\mathrm{UV}$ were always above $30 \mathrm{fps}$ and could reach $80 \mathrm{fps}$ depending on the number of focus points. 3D-UV regularly were recorded as transverse parallel planes.

Acquisition of 3D-UV and quality assessment

\section{Study population, recruitment of models}

With informed consent, four healthy models $(n=2$ females, age 26 and 24 years; and 2 males, age 17 and 24 years) served to obtain normal sonoanatomy. Models had no co-morbidity and no pathologic finding, and the ultrasound exam of respective body areas was recorded. The staff of the university hospital were recruited by announcements. Inclusion criteria were: age above 18 years and a body mass index (BMI) of less than 30; exclusion criteria were: age below 18 years and BMI over 30 .

\section{Setting, location and team}

Data were collected at the Hospital of the University of Frankfurt am Main. For recordings within a standardized environment, a room of the emergency department was equipped with the complete ultrasound simulator system and regularly maintained by two staff technicians. One medical doctor recorded 3D-UV in a team with the technician on a model. The medical doctor is an expert practitioner on basic and intermediate UGRA level blocks [1]. The staff technician was educated by the manufacturer to perform ultrasound 3D-UV recordings. Recordings were viewed later and selected by eye-balling, whenever a target anatomical site was clearly identified. Those 3D-UV were collected and stored in a case library on the ultrasound simulation system and combined to 3D-multivolumes (3D-MV).

On every model, respective regions of the right body area were scanned. Recordings were also obtained as 2D-B-Mode images or clips by the ultrasound device. After recording and storing, 3D-UV were positioned with an electromagnetic tracking system into a soft foam mannequin to the corresponding anatomical sites originally obtained on the model. Using developer software, single B-Mode 3D-UV were combined into 3D-MV. The advantage of $3 \mathrm{D}-\mathrm{MV}$ is that one single case of the 

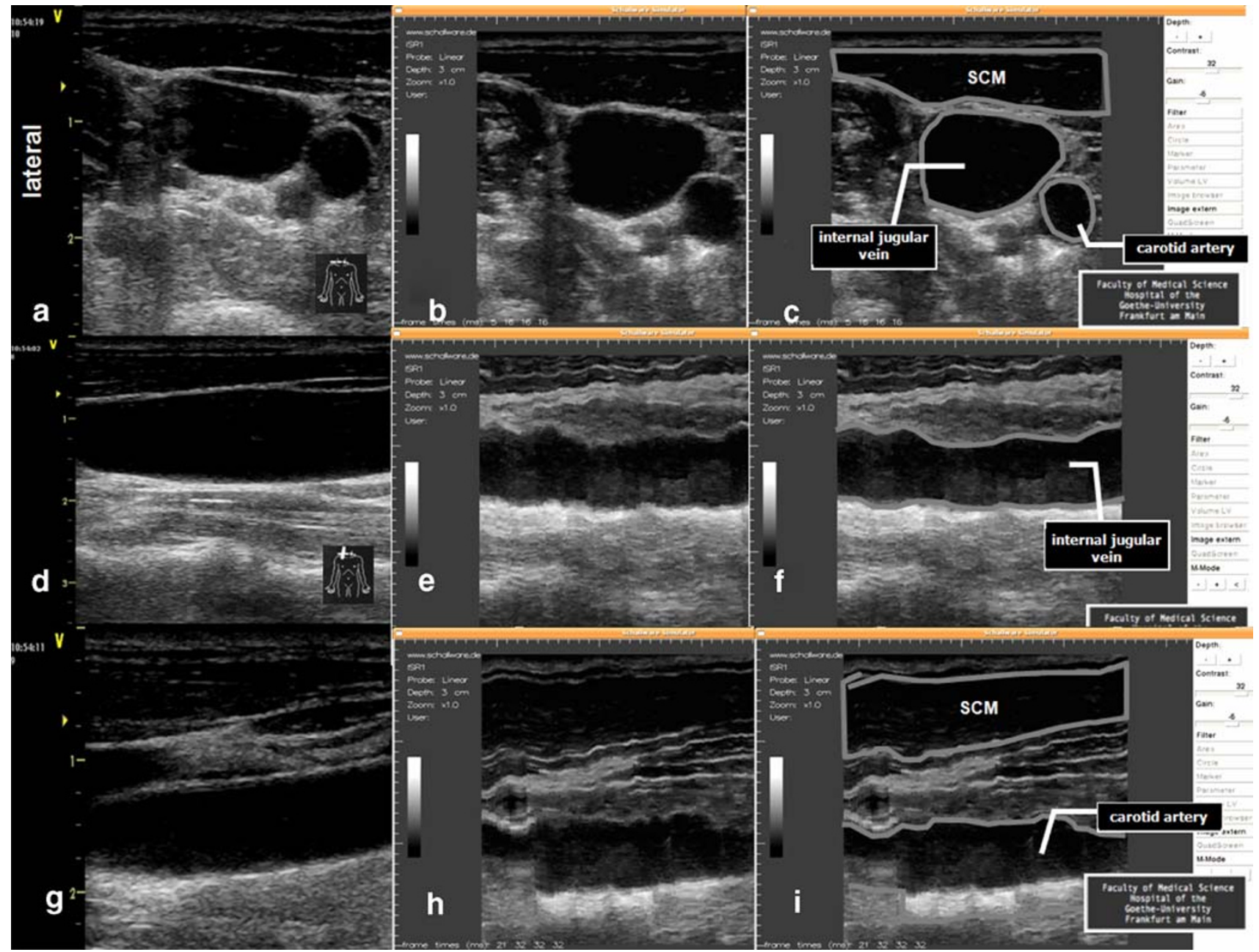

Fig. 1 Original and reconstruction of planes in ultrasound simulation of vessels in the carotid region, $S C M$, sternocleidomastoid muscle. $3 \mathrm{D}$-recordings were performed in continuous parallel movements of transverse B-Mode scans using a free-hand use electromagnetic tracking system connected with an ultrasound device (Vivid-i, reg. TM by GE Healthcare Ultrasound). Planes were obtained by real time examination on a dummy after positioning of the 3D ultrasound volume into the torso. a, d, $\mathbf{g}$ Original B-Mode planes from the

ultrasound simulation can combine multiple 3D-UV and allows more examinations and workflow without changing or interrupting by the operator. This system allows operators to examine any position in similar way as using an ultrasound device and can be performed near-real close to the examination of a model or patient. Image adaptations can be made related to depth, contrast and gain. For documentation, a freeze function and storing of screenshots are available which can be evaluated by trainers.

Single recordings of 3D-UV were continuous transversal views along the body axis ("horizontal sweep"). Regions included carotid trigonum for jugular vein, carotid artery and soft tissues or bone, interscalene region to record ultrasound device (short axis, SAX): $\mathbf{d}, \mathbf{g}$ (long axis, LAX). b, c, e, f, h, i Planes generated by the Ultrasound Simulator "Reslice" (reg. TM by Schallware $\mathrm{GmbH}$, Berlin, Germany): b, c SAX planes of the carotid region. e, f LAX planes of the internal jugular vein. $\mathbf{h}, \mathbf{i}$ LAX planes of the common carotid artery. As only transverse (SAX) scans were recorded with frame rates over $30 \mathrm{fps}$, reconstructed LAX planes all contain the original pulsation of the vessel. The wall in the LAX thus appears as "see-saw" artefacts

brachial plexus, peripheral nerves distal to the axilla (median, ulnar, radial and musculocutaneous), inguinal region for femoral vessels and femoral nerve and popliteal crease for distal sciatic nerve and branches (popliteal and tibial branch).

\section{Evaluation of reslices and statistical methods}

Neither a reference method for UGRA was described nor does a reference ultrasound machine or setting serve as a gold standard. High-resolution ultrasound technology using high frequency ultrasound ( $>10 \mathrm{MHz})$ is considered to be somewhat a major prerequisite. Therefore, no blinding for the test results of 3D-UV was included. 
Original SAX and long axis (LAX) B-Mode planes were compared with the reconstructed planes (=reslices) by the expert practitioner who recorded the 3D-UV. Image quality was assessed by eye-balling always during repositioning. This was done qualitatively for descriptive statistic purposes only. Reslices were compared qualitatively to original recordings of $2 \mathrm{D}$ clips or $2 \mathrm{D}$ images stored on the ultrasound device. Additionally, quality of SAX view was assessed by obtaining an image of a recorded 3D-UV target structure and rotation of $90^{\circ}$, resulting in a longitudinal slice (LAX) of the reconstructed 3D-UV. These LAX 3D-UV reslices were also compared to original LAX images of the model. Comparison resulted only in selecting or discarding of the recordings. A further visual estimation test for correlation analysis between original images and 3D-UV was not planned. Test reproducibility was controlled by repeated recording of an anatomical site up to ten times. A quantitative test reproducibility was not planned. From these repeated recordings, 3D-UV were chosen for further analysis.
This study and project plan was granted by the local review board of the University of Frankfurt am Main.

\section{Results}

One hundred and eight recordings of 3D-UV including vessels of carotid region $(n=22)$ and peripheral nerves of interscalene (31), axillary (28), groin (8) and distal sciatic (19) were obtained from four models. A total of $37 / 108$ (34\%) recordings had to be discarded because of lack of showing the target (3), indeterminate results (3), poor quality (8), unintended model movement (2) of lack of recording of files (18). Study was performed between August 2008 and March 2009.

Visual presentation of reconstructed SAX volumes of vessels and peripheral nerves revealed almost comparable quality to the original B-Mode scans of the model examination (Figs. 1, 2, 3, 4, 5). Importantly, ultrasonographic nerve structure appearance derived from high-resolution ultrasound recordings could be preserved in reslices of

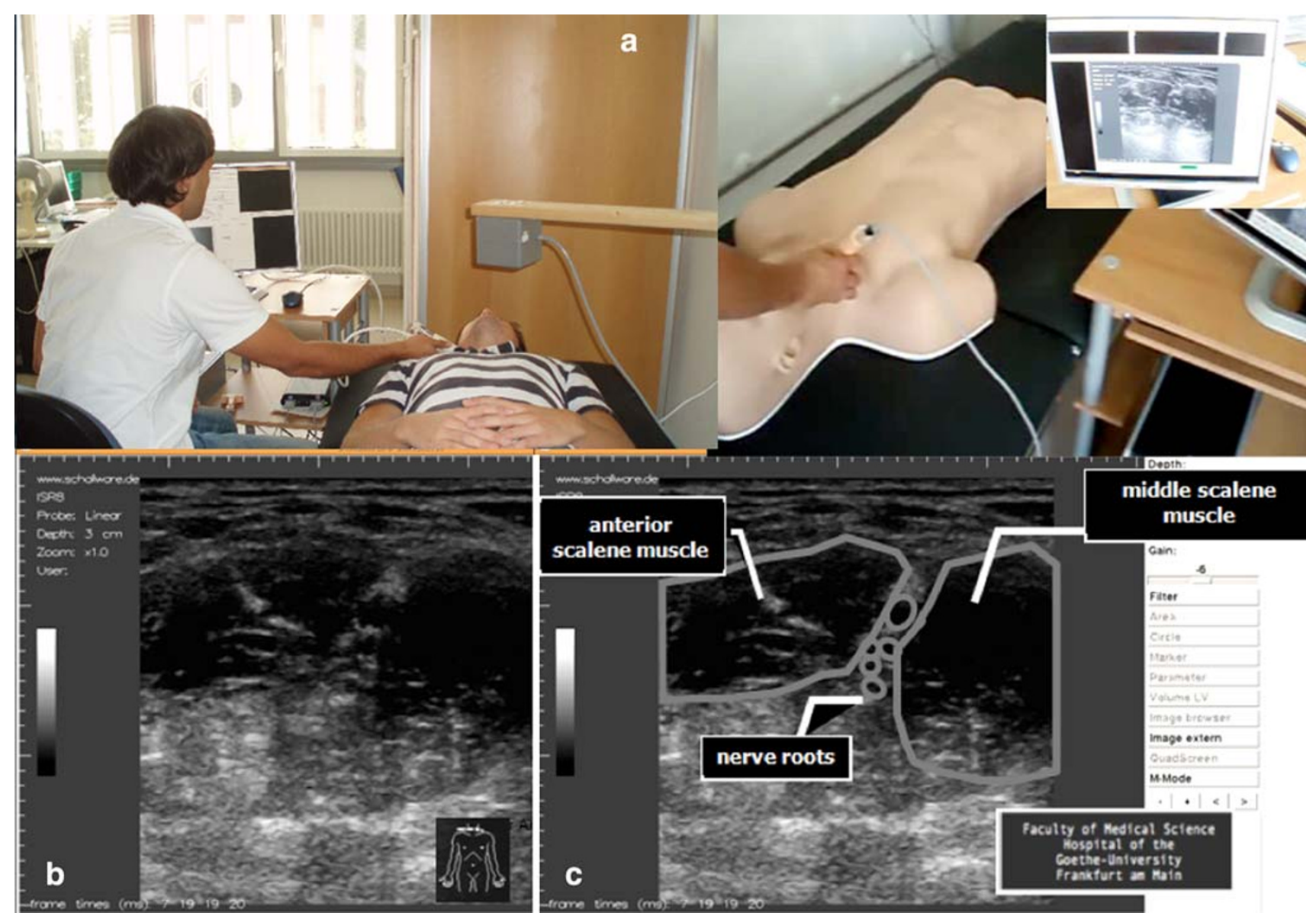

Fig. 2 a Setting of ultrasound simulation recording system (upper left) and examination of the interscalene region (upper right). b, $\mathbf{c}$ Reconstructed plane from the ultrasound simulator for interscalene approach of brachial plexus 


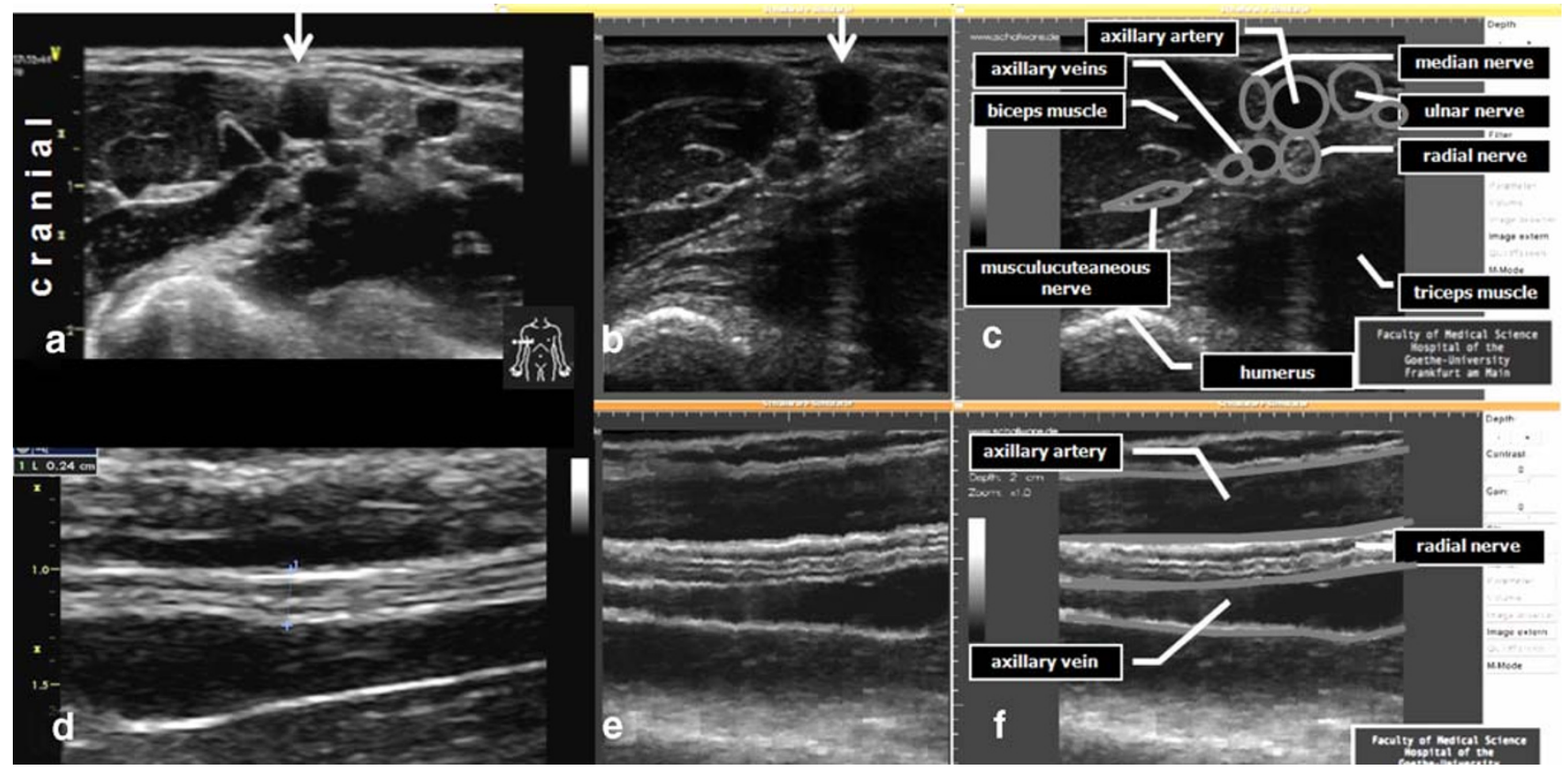

Fig. 3 a, d Original planes of axillary region. Arrow marks axillary artery. Reconstructed planes (b, c SAX; e, f LAX). Note that radial nerve in LAX plane shows typical hyperechoic and hypoechogenic parts as described for nerve ultrasonic appearance with high resolution technology. This ultrastructure could be preserved completely in the reconstructed plane and real time examination with the ultrasound simulator $(\mathbf{e}, \mathbf{f})$
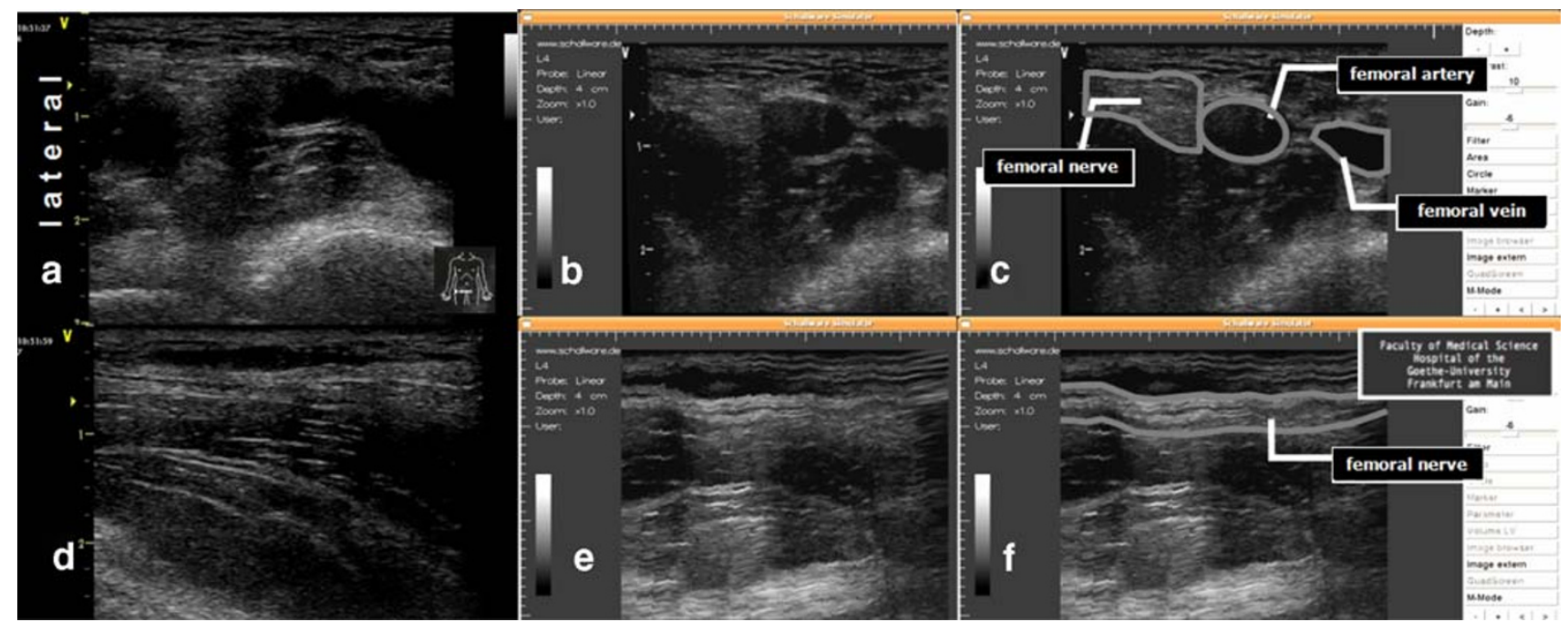

Fig. 4 Original $(\mathbf{a}, \mathbf{d})$ and reconstructed planes $(\mathbf{b}, \mathbf{c}, \mathbf{e}, \mathbf{f})$ of the right groin showing femoral nerve and vessels in SAX (a-c) and LAX $(\mathbf{d}-\mathbf{f})$ planes

3D-UV and also in part in LAX (Figs. 3, 4, 5). Moreover, muscle, fascia and bone were reconstructed comparable to the original image (Figs. 3, 4, 5).

As expected, reconstructed LAX view of carotid artery and internal jugular vein in ultrasound simulator image planes showed good quality, but were partially dependent on direct pulsation. Vessel wall thus appeared as "seesaw" artefact (Fig. 1). However, pulsation of smaller axillary vessels did hardly affect LAX image reconstruction of radial nerve (Fig. 3) and median or ulnar nerve (not shown).

\section{Discussion}

This study serves as basic research for ultrasound simulation of peripheral nerves. Regarding clinical application of our findings, ultrasound simulation can serve as innovative 

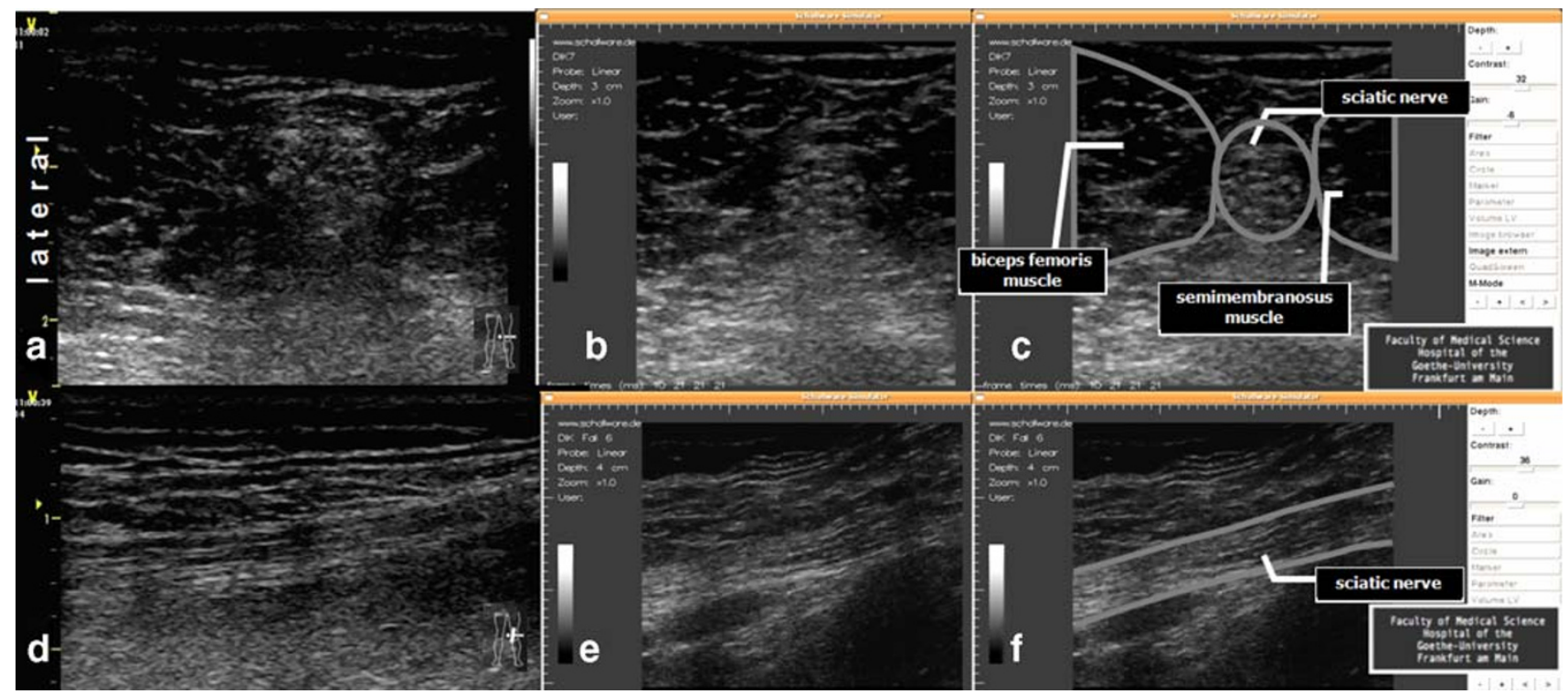

Fig. 5 Original $(\mathbf{a}, \mathbf{d})$ and reconstructed planes $(\mathbf{b}, \mathbf{c}, \mathbf{e}, \mathbf{f})$ of the right dorsal distal thigh showing sciatic nerve (SN) in SAX (a-c) and LAX (d-f) planes. SN 3D volumes were recorded from the popliteal crease
$10 \mathrm{~cm}$ cephalad, immediately before branching into the peroneal and tibial branch method in education and training [3-5] and preparing staff before handling specialized procedures in UGRA. Here, we show that ultrasound of vessels and peripheral nerves can be reconstructed from 3D-UV with preservation at highresolution ultrasonographic appearance of nervous echo texture [6] by means of a simple electromagnetically tracking system [5]. Such 3D-UV are well suited to study sonoanatomy, learn handling and movement of ultrasound probe, plane interpretation for UGRA and of sonoanatomic landmark structures for specific block techniques. Additionally, this system can allow training needle positioning in both out of plane and in-plane approaches and needle movement on the basis of the 3D-MV without model or patients, which is an important functional aspect for training of UGRA.

Next steps of development should address to obtain a series of cases to show better anatomical variations. These are well known for the axillary region $[1,7]$. Of further interest could be recordings of 3D-MV with pathology such as enlarged axillary or cervical lymph nodes, neurinoma of peripheral nerves or plexus, calcification of muscle or haematoma, oedema, which are rare findings within UGRA.

\section{Limitations}

There are many limitations related to this ultrasound technology. One limitation is the positioning of the 3D-UV or 3D-MV, because of the anatomical differences between various models and a standard sized ultrasound dummy have to be realized. Also, at this stage no probe compression test to distinguish between vein and artery or direct estimation of pulsation of arteries can be applied to the dummy. Further, colour Doppler can be recorded and reproduced in 3D-UV. Although mimicking needle insertion by software-guided needle-dummy visualization into the region of interest by operator is available, neither a real needle can be inserted into the dummy nor the spread of a local anaesthetic can be visualized with this system yet. However, while further development, real-time 3D ultrasound volumes (4D) may be able to demonstrate this important teaching task in the future.

\section{Conclusion}

Our data provide a new proof of concept for ultrasound simulation of peripheral nerves in 3D-ultrasound multivolumes based on high-resolution ultrasound technology. It has also the ability to provide pathologic findings and may serve in the future as a powerful method for training in regional anaesthesia and critical care ultrasound.

Acknowledgments We gratefully acknowledge the faculty of medical science and Dean Prof. F. Nürnberger for providing the ultrasound simulation system and grant to the interdisciplinary team of TSR. Special thanks to Gernot Jehle, Schallware GmbH, Berlin, Germany, Dirk Beermann as well as Monika Krammer, GE Healthcare Ultrasound, Solingen, Germany for technical support. We are also indebted to the models and technical assistance of T. Telles and M. Dreger. There was no financial support by the companies mentioned in this paper. 
Conflict of interest statement No conflict of interest related to the publication of this manuscript.

\section{References}

1. Sites BD, Chan VW, Neal JM et al (2009) The American Society of Regional Anesthesia and Pain Medicine and the European Society Of Regional Anaesthesia and Pain Therapy Joint Committee recommendations for education and training in ultrasoundguided regional anesthesia. Reg Anesth Pain Med 34(1):40-66

2. Xu D, Abbas S, Chan VW (2005) Ultrasound phantom for handson practice. Reg Anesth Pain Med 30(6):593-594
3. Knudson M, Sisley AC (2000) Training residents using simulation technology: experience with ultrasound for trauma. J Trauma 48:659-665

4. Salen P, O'Connor R, Passarello B et al (2001) FAST education: a comparison of teaching models for trauma sonography. J Emerg Med 20(4):421-425

5. Terkamp C, Kirchner G, Wedemeyer J et al (2003) Simulation of abdomen sonography. Evaluation of a new ultrasound simulator. Ultraschall Med 24(4):231-232

6. Silvestri E, Martinoli C, Derchi LE et al (1995) Echotexture of peripheral nerves: correlation between US and histologic findings and criteria to differentiate tendons. Radiology 197(1):291-296

7. Retzl G, Kapral S, Greher M, Mauritz W (2001) US findings of the axillary part of the brachial plexus. Anesth Analg 92:1271-1275 